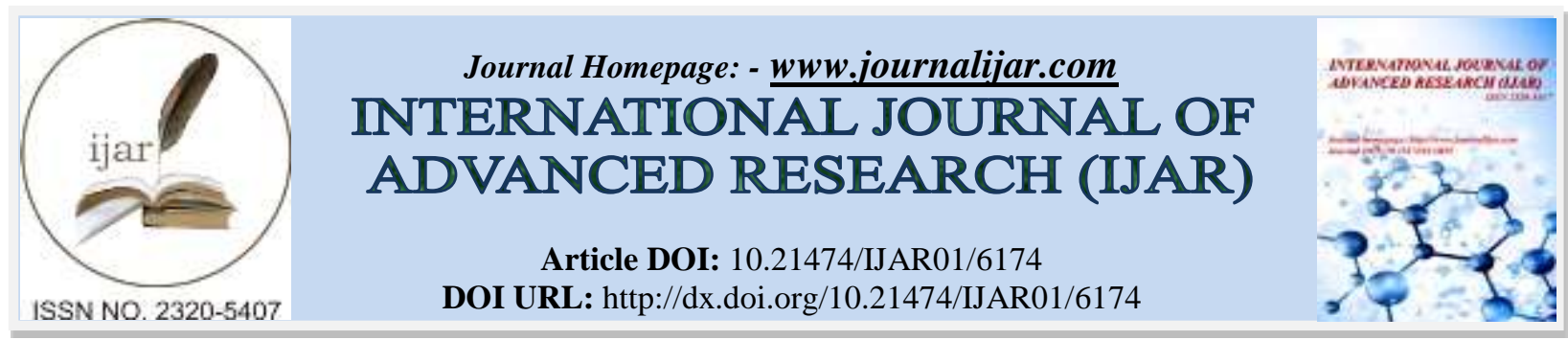

RESEARCH ARTICLE

\title{
UTILITY OF ANALYTICAL APPROACH FOR VALUATION AND CHARACTERIZATION OF USER BASED NETWORK MONITORING.
}

1. Research Scholar, Department of Computer Science.

Sarita $^{1}$ and Vijay Pal Singh ${ }^{2}$.

2. Assistant Professor, OPJS University, Churu, Rajasthan.

\section{Manuscript Info}

-

Manuscript History

Received: 25 October 2017

Final Accepted: 27 November 2017

Published: December 2017

Keywords:-

Network monitoring, Characterization of

User Based Network.

\begin{abstract}
Precise activity classification is of fundamental note worthiness to a few other system sports, from wellbeing following to bookkeeping, and from charming of transporter to giving administrators valuable conjectures for protracted day and age provisioning. We take after a Naïve Bayes estimator to sort movement by utility. Interestingly, our sketches underwrite helpful ordered group records, the utilization of it as contribution to a regulated Naive Bayes estimator. on this paper we delineate the unreasonable phase of exactness achievable with the Naive Bayes estimator. We comparably represent the ventured forward precision of sensitive varieties of this estimator. Indeed, even as our strategy makes utilization of preparing realities, with classifications gotten from parcel content, the majority of our preparation and experimenting with changed into finished the use of header-inferred discriminators. We underline this as an intense part of our strategy: the utilization of tests of understood activity to permit the order of guests the use of generally accessible data all alone. On-line site guests grouping stays of long time interest to the systems administration arrange. It serves on the grounds that the contribution for sensible answers, for example, organizes following, magnificent of-transporter and interruption recognition. A fundamental issue of this strategy is that it wires actualities from glide estimations taken over the span of a system. We take after the subspace technique to 3 unique assortments of tested accept circumstances for what they are activity in a substantial instructional group. Multivariate time arrangement of byte checks, parcel numbers, and IP-drift tallies. We show that each movement kind brings into mindfulness a phenomenal arrangement of irregularities through the subspace technique.

Copy Right, IJAR, 2017,. All rights reserved.
\end{abstract}

\section{Introduction:-}

General strategies for recognizing oddities in arrange activity is an imperative, unsolved issue. In main, it ought to be conceivable to watch most inconsistency sorts by assessing activity streams. In any case, to date, there has been little advance on extricating the scope of data introduce in the entire arrangement of activity streams in a system. There are numerous great explanations behind this: activity streams exhibit numerous conceivable sorts of system movement; the arrangement of all streams involves a high-dimensional space; and gathering all movement streams 
is exceptionally asset serious. Regardless, the undeniably across the board utilization of apparatuses, for example, Net Flow [4] by ISPs make it practical to think about techniques for productively gathering and adequately dissecting movement streams. Advances in correspondence innovation and the expansion of lightweight, hand-held gadgets with worked in, fast radio get to are making remote access to the Internet the normal case as opposed to a special case. Remote LAN establishments in view of IEEE 802.11 [8] innovation are rising as an alluring answer for giving system network in organizations and colleges, and out in the open spots like meeting settings, air terminals, shopping centers, and so on - places where people spend a lot of their time outside of home and work. Notwithstanding the accommodation of courageous systems administration, contemporary remote LANs gives moderately high information availability at $11 \mathrm{Mb} / \mathrm{s}$ and are anything but difficult to convey openly settings. As a major aspect of a bigger research extend, we have been investigating issues in executing and conveying open territory remote systems, and investigating improvements for enhancing their execution [1]. To assess and approve the systems that we are creating, we think of it as basic to utilize practical workloads of client conduct and remote system execution to settle on outline choices and exchange offs. Be that as it may, since open remote LANs have just as of late turned out to be broadly conveyed, such workload portrayals are rare. Beginning investigations of remote systems have investigated low level mistake models and RF flag qualities [5], establishment and support issues of a grounds remote system [3], client versatility in a low-transfer speed metropolitan zone organize [8],

The Internet is developing towards a huge, pervasive foundation, supporting an undeniably colossal market of information correspondence and advanced media and delivering trillions of dollars of income every year. The information transmission is administered by straightforward end-to-end transmission conventions, for example, TCP and UDP, without effective checking, reviewing and canny control over the activity, yet the achievement of the Internet has prompted the development of an apparently uncountable assortment of utilizations. Alongside the improvement and development of the applications on the Internet, a proficient application order plot is profoundly attractive to help different arrangements, for example, propelled organize checking, organize asset administration, abnormality discovery, application-particular systems and system examining exercises. In addition, the applicationlevel information of the Internet is to a great degree helpful for the individuals who embarked to show Internet activity or to explore the long haul changes and necessities for the Internet. The Internet movement, on a fundamental level, is the result of a complex multi factor framework including a scope of systems, hosts, applications and distinctive individuals intently interfacing with each other. The unpredictability is constantly expanding as individuals continue creating an immense assortment of system applications and application layer conventions that, from multiple points of view, break the customary suppositions. In light of watched activity designs, we built up a grouping plan giving close continuous characterization of up to $99.8 \%$ of movement, utilizing behavioral elements and $\mathrm{C} 4.5$ choice tree calculation [4].

This approach is on a very basic level not quite the same as customary activity order approaches in that:

1. It does never again depend on port numbers. Moreover, we assume no prior data about port-programming mapping in our approach.

2. Our method does not require the examination of site guest's payload.

3. The behavioral components, e.g. appropriation of the size of bundles, TCP window length, TCP hail bits and parcel rules, are gotten from the parcel headers, a similar wellspring of actualities this is anticipated to be utilized by the switches of the web.

\section{Related Work:-}

In late writing, a wide range of strategies have been acquainted with take care of the movement order issue. The larger part of current characterization approaches still depends on the data of host port numbers, IPs and marks for arrangement and interruption recognition, for example, light-weighted interruption location frameworks including Bro [5] and Snort [6]. Moore and Zuev in [7] exhibited a factual way to deal with group the movement into various sorts of administrations. A gullible Bayes classifier, consolidated with part estimation and a connection based sifting calculation was utilized to take care of the arrangement issue on disconnected TCP follows. The subsequent exactness, up to $96 \%$ (which corrupts to $93 \%$ following 8 months), exhibited the discriminative energy of a blend of 10 stream conduct highlights, with an unsophisticated machine learning component. Williams et al. in [8] completed an exact investigation of looking at five broadly used machine learning calculations to order Internet activity. Among these calculations, AdaBoost+C4.5 accomplished the most noteworthy exactness in their outcomes. This fills in as a manual for calculations, however their list of capabilities utilized is generally unsophisticated, containing just bundle lengths, add up to bytes, add up to parcels, between entry times, stream span and convention. In light of the instrument in this work, [9] additionally proceeded onward to order diversion activity with a perception window 
of close to 25 parcels, which is likewise anticipating constant characterization. Bernaille et al. exhibited a way to deal with distinguish applications utilizing begin of-stream data in [10].

The creators used the parcel size and bearing of the initial 4 information bundles in each stream as the components with which they prepared Gaussian and Hidden Markov Models individually. These models got $98.7 \%$ general exactness when helped by an extended port number rundown, and $93.7 \%$ general precision utilizing basic forecast heuristics. It is significant that this work just recovered a little measure of data from the streams. The creators additionally particular their work to the recognizable proof of encoded activity in [1]. Another factual finger printing system was proposed in [2]. The data they utilize incorporate size of the IP bundles, between landing time and the request of parcels seen on the connection. Various different works endeavored a similar issue with various machine learning systems, for example, grouping [3] [4], LDA and k-NN [3]. Karagiannis et al. [5] considered multi-level conduct of the activity, for example, examining communication between has, convention use and per-stream normal parcel estimate. Their outcomes demonstrate a capacity of ordering 80\%-90\% of the movement with $95 \%$ exactness. In their current work [1] they exhibited an intriguing examination to profile the clients movement and practices, and to dissect the dynamic attributes of the host practices. From the outcomes as far as exactness we may declare that the blend of few stream conduct highlights as of now has solid discriminative energy to separate administrations or system applications. For non-abnormal activity and inside a little size of time (e.g. in the extent of inside a day), these arrangement instruments can be impressively encouraging (with an exactness up to 98\% and continuous potential appeared). Be that as it may, the outcomes in the past stayed deficient. Some real concerns and open issues included:

1. The exactness is as yet inadequate. For universally useful arrangement, the volume of Internet activity is immense and is ruled by a couple of significant movement classes (Web perusing as far as streams, or Peer-2Peer and FTP regarding bytes or parcels). As far as organization, the significant movement is similarly simple to separate. Be that as it may, those hard ones, for example, typified, equivocal, non-standard, abused or abnormal activity would just contain a not huge extent. A general mistake rate of a few percent would mean either the real movement is not effectively classified; or the hard protests can't be classified.

2. Insufficient comprehension has been assessed which elements can be utilized and how to utilize them. Further, small comprehension between the exactness and the utilization of various sorts of components (port numbers, IP Addresses, stream conduct) has been displayed previously.

3. Limited practicability. Outstandingly, ongoing usage is exceedingly attractive, yet lacking work has been done to demonstrate the possibility and the framework execution.

4. Inability to rapidly find and effectively recognize basic streams, for example, interruptions and system oddities. Interruption location frameworks would in a perfect world require zero false-negative rate and low-inactivity ID of noxious activity.

5. The inadequacy of information. Numerous past works chose their order question from a couple of utilizations, for example, web-program, email, FTP or sight and sound applications, which cannot speak to completely the activity examples of the entire Internet.

\section{Analytic Machine Learning Approach:-}

We utilize a semi-automated gadget considering method to develop the classifier which orders the web guests into application directions. a chain of components are actualized to pick the capacity set, the sort set of tenets and the size of analysis window sooner than the last classifier is developed [5]. Leading the full activity blend is part into classifiable items. on this paper, the basic protest of our order gadget is a TCP stream, depicted as a bi-directional meeting between two hosts with a similar five-tuple have IP, benefactor IP, have Port, buyer Port and timestamp of the primary bundle. The server and the supporter of a buoy are resolved fundamentally in view of proclamation of SYN parcel. Two genuine strains are utilized to infer and assess our approach. Rich arrangements of 248 stream abilities are collected from the earliest starting point of man or lady organize streams with select articulation window sizes. the utilization of this realities, we can take after trademark determination calculations to find a superb subset of the elements, to legitimize the class calculation. At some point or another, we instruct a model upon this determination subset, and take after this variant to arrange obscure streams. Fig. 1 demonstrates how restrictive assortments of offerings grandstand particular lead in establishment each of two abilities: 1) change of general bytes in parcels (benefactor to server) through the general assortment of bytes dispatched in preparatory window (client to server) and a couple of) recollect of bundles with Push bit set in TCP header (server to customer) by negligible area length (buyer to server). You'll watch that it is relevant to separate between the movement streams of every polish, the use of a total of those capacities [2]. 
Our trial measurements comprises of two back to back week-days of net site guests with an eight month c programming dialect. The follows had been accumulated utilizing a high pace observing holder [8] built up between an exploration grounds and the web. The grounds is an investigations office with around 1,000 faculty and is appended to the web through a total duplex Gigabit Ethernet hyperlink. The datasets, Day1 and Day2, for example, TCP movement handiest, are chosen from a set unmistakable in [9]. Each drift inside the two datasets progressed toward becoming hand-classified utilizing a substance material-based component into one of the 10 applications directions. We cleared out various TCP site guests inside the datasets unconsidered: the ones we haven't obvious their begin of the drift (by and large the ones are with extremely long period) and garbage streams. The following lines incorporate 31 GBytes and 42 million bundles in 377 thousand TCP streams in Day1, and 28 GBytes and 35 million parcels in one hundred seventy five thousand TCP streams in Day2. thusly, a respectably confounded mix of projects exists inside the activity, as demonstrated in work area I. Watch that for some activity lessons comprehensive of Multimedia, administrations, computer games and ambushes, an additional amount of movement may likewise utilized diverse transport layer conventions alongside UDP.
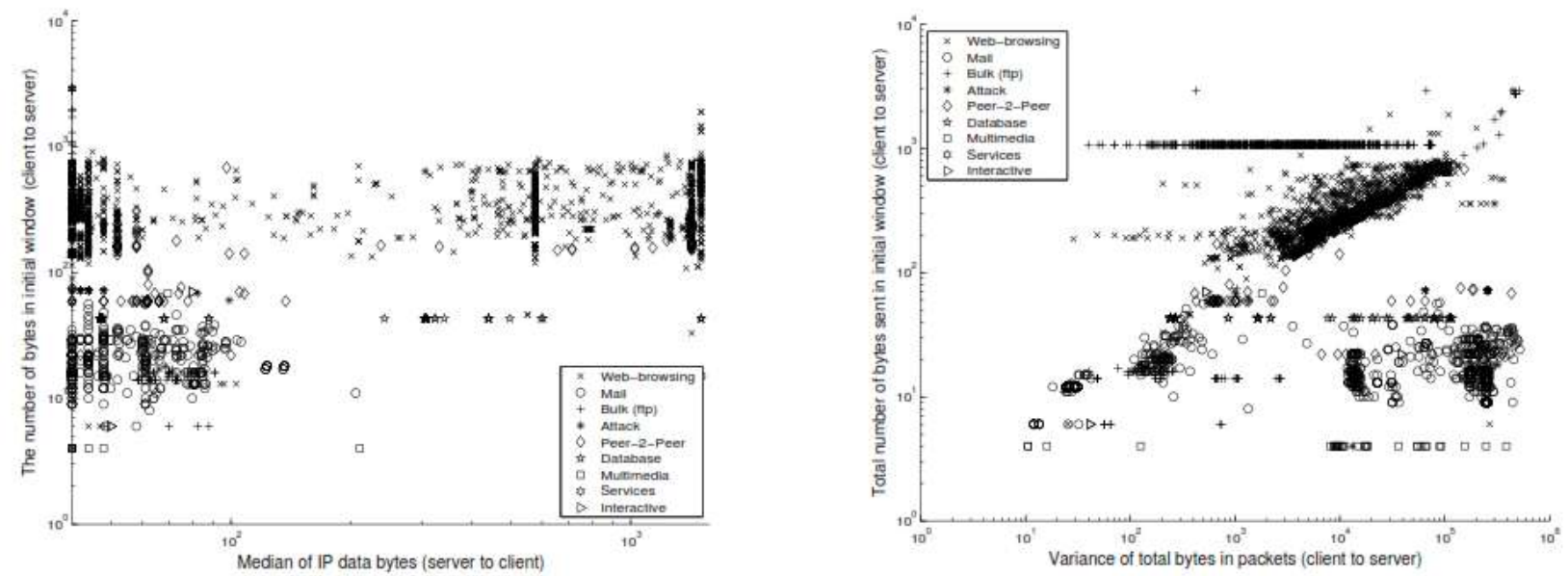

Figure-1:- Random selected samples

Our online activity grouping technique was begun in past disconnected approach where the components were gathered from finish TCP streams [7] [2]. Nonetheless, in the event that we played out our investigation of streams disconnected, the practicability of such a framework would be particularly constrained to just explanatory and evaluating purposes. For a considerably more extensive application prospect, we proceeded onward to research the issues in principle, with a specific end goal to all the more precisely order a question, it would require gathering more data (entropy) for characterization. Be that as it may, gathering more data, for instance: more parcels or a bigger number of elements may present higher dormancy and higher cost in both calculation and memory use. For the activity grouping framework to be worked at close continuous with an extensive throughput, we should catch a proper, modest number of elements, and from few parcels and a constrained span, instead of from a total stream. That is to say, keeping in mind the end goal to pick up the constant quality, a measure of data must be yielded from the entire stream objects which in principle would bring about some level of corruption in exactness. Along these lines, the exchange off between exactness, dormancy and throughput turns into our preferred key of subset of components and size of the perception window and the grouping calculation.

Our entire list of capabilities contains 248 unique elements as nitty gritty in [9], each of which has fluctuated dispersion in the datasets and has related with it distinctive accumulation/calculation costs. Presently what we need is to discover a subset of this arrangement of elements inside an upper bound of cost however containing adequate data that prompts the coveted exactness. All in all, we confine the cost by decreasing the quantity of elements in the list of capabilities, using a connection based sifting technique. The yield of such technique is an around best subset of elements. Our component determination technique is as per the following: right off the bat, Day1 dataset is separated into 10 distinct sections each speaking to a volume of activity at various hours of the day; at that point relationship based sifting is connected to every passage. We watch that these component subsets chosen by the calculation have respectably great strength, and we physically picked 10 conduct highlights which show up in no less than $1 / 3$ of the subsets, and every subset would at any rate contain $1 / 3$ of these elements. The goal of this 
foundation is to search for a most ideal list of capabilities which can be more steady and autonomous to the state of the end to-end connect. The subsequent components are altogether relied upon the applications on the end-has. It reflects this thought. Table II records these components, and also the data and many-sided quality properties of these elements.

\section{Classification Algorithm for proposed approach:-}

Concerning the arrangement calculation, we used Weka [6] toolbox to analyze between various calculations. In our approach, C4.5 choice tree displays most astounding precision (97.824\%) among every one of the calculations. AdaBoost [22]+C4.5 likewise surpassed 98.9\%, while Logitboost, JRip, Nave Bayes Tree and Bayesian Neural Network additionally accomplished an exactness of over 99\%. Also, C4.5 choice tree has the most reduced testing unpredictability among these calculations. In Table III, the execution of C4.5 and Adaboost+C4.5 are indicated contrasted and the Nave Bayes technique which was utilized as a part of [8]. Note that the approach in [7] did not fuse numerous standard execution upgrades for Naive Bayes technique is a most pessimistic scenario bound. A comparative examination was seen in [8] in view of an easier arrangement of finish stream highlights.

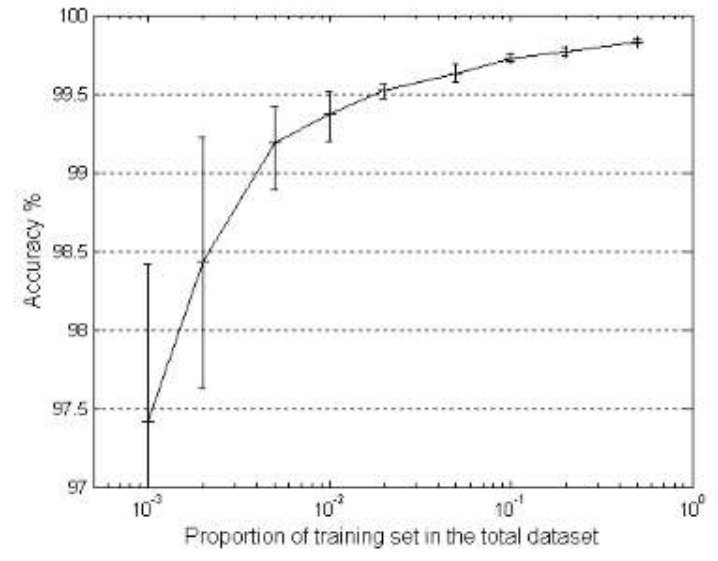

(a) $\mathrm{C} 4.5$

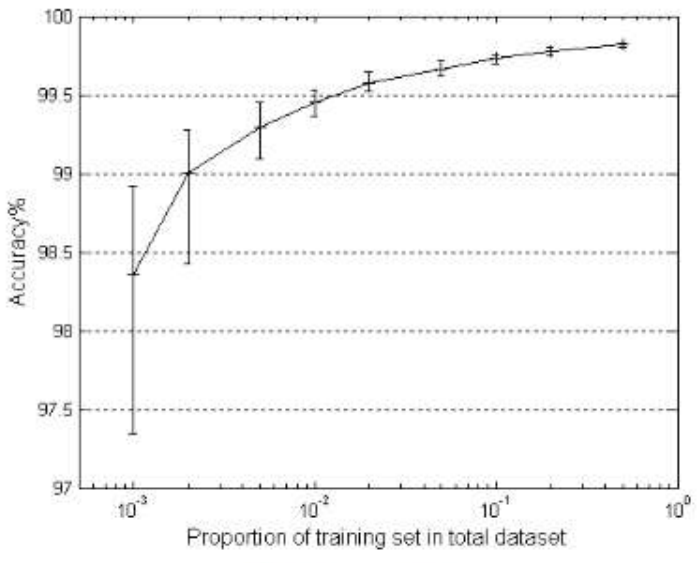

(b) Adaboost+C4.5

Figure-2:- Average accuracy with compare size of training set

\section{Conclusion:-}

Inside the net there's a continually expanding amount and kind of activity. Provoked by method for a longing to see the bundles of the web, on this paper we display a gadget acing strategy for group website guests classification fundamentally in view of guest's conduct. By method for accumulating a little scope of capacities, from a little scope of parcels or a concise time of a site guests stream, our approach can offer legitimately adjust on the general execution: the precision, throughput and idleness. It has various points of interest over customary port and mark basically based frameworks, which incorporate the ability to see encoded streams or streams the utilization of irregular ports, and the capacity to handle earlier obscure bundles. At last, it has demonstrated exceptionally encouraging attainability for sensible utilize. in the back of this work, we have one clear aim which is to apply this sort plan to reasonable bundles. For this, there are as yet various ranges in which future work ought to likewise legitimize the possibility and appropriateness. We comprehend that the test of finding the astounding conceivable mix of capacities and strategy remains perceptibly utility-exact and merits additionally inquire about. long haul fleeting parity and spatial adjust remains an essential topic to set up the more extensive pertinence of the approach yet will require all the more preparing insights sets. 


\section{Reference:-}

1. Manuel Crotti, Maurizio Dusi, Francesco Gringoli, and Luca Salgarelli. Traffic classification through simple statistical fingerprinting. SIGCOMM Computer Communication Review, January 2007.

2. J. Erman, M. Arlitt, and A. Mahanti. Traffic classification using clustering algorithms. In Proceedings of the 2006 SIGCOMM workshop on mining network data (MineNet '06), September 2006.

3. K. Gopalratnam, S. Basu, J. Dunagan, and H. Wang. Automatically extracting fields from unknown network protocols. In First Workshop on Tackling Computer Systems Problems with Machine Learning Techniques (SysML06), June 2006.

4. Thomas Karagiannis, Konstantina Papagiannaki, and Michalis Faloutsos. Blinc: multilevel traffic classification in the dark. In Proceedings of ACM SIGCOMM 2005, pages 229-240, 2005.

5. Thomas Karagiannis, Konstantina Papagiannaki, Nina Taft, and Michalis Faloutsos. Profiling the end host. In Passive and Active Measurement Conference 2007 (PAM'07), April 2007.

6. E. Anderson and M. Arlitt, "Full packet capture and offline analysis on 1 and $10 \mathrm{gb} / \mathrm{s}$ networks," Technical Report, HPL-2006-156 20061106, HP Labs, Tech. Rep., 2006.

7. R. Prasad, M. Jain, and C. Dovrolis, "Effects of interrupt coalescence on network measurements," in The 5 annual Passive \& Active Measurement Workshop, (PAM 2004), Antibes, France, April 2004.

8. L. Deri, "Improving passive packet capture: Beyond device polling," in Proceedings of SANE 2004, 2004.

9. [9] K. Mackenzie, W. Shi, A. Mcdonald, and I. Ganev, "An Intel IXP1200based network interface," in Proceedings of the Workshop on Novel Uses of System Area Networks at HPCA (SAN-2 2003), 2003.

10. T. Nguyen, M. Cristea, W. de Bruijn, and H. Bos, "Scalable network monitors for high- speed links: a bottomup approach," in Proceedings IEEE Workshop on IP Operations and Management, 2004, Beijing, China, October 2004, pp. 16-22. 